\title{
DESCOMPOSICIÓN DE HOJARASCA EN UN TORRENTE ÁCIDO DE ITALIA CENTRAL
}

\author{
Jose' Giancarlo Morgana y Susanna Prato \\ ENEA Casaccia. Area Energia Ambiente e Salute. \\ Via Anguillarese, 301. S. Mana di Galeria, 00060 Roma (Italia).
}

Palabras clave: Ríos, hojarasca, acidificación, comunidades de macroinvertebrados

\section{ABSTRACT \\ LEAF PACKS BREAKDOWN IN AN ACID STREAM OF CENTRAL ITALY}

The aim of this work is to analyse the decomposition trend of Castanea sativa and Alnus glutinosa leaves in an acid stream of Central Italy: the Fosso Caldara. Breakdown rates were measured by confining leaf packs in three sampling sites along a gradient of water acidity and calculating their loss of biomass after 7, 21, 35, 56, 77 and 105 days of immersion. The processing of both kinds of leaf packs was compared. It is pointed out that high water acidity reduces the breakdown rates and affects the macroinvertebrate communities living on detritus of each sampling site reducing their taxa richness.

\section{INTRODUCCIÓN}

Algunos estudios científicos sobre la acidificación de los ríos han tenido como finalidad poner en evidencia los efectos de ésta sobre los procesos que ocurren en el ecosistema (GROOM \& HILDREW, 1989). La acidificación de los ríos causa una alteración general de la estructura de las comunidades lóticas que comporta, por ejemplo, la reducción de la diversidad de la comunidad de macroinvertebrados bentónicos (TOWNSEND $\boldsymbol{e t}$ al., 1983; KIRBY, 1992). Dicho empobrecimiento de las comunidades animales se debe sobre todo a las condiciones químicas asociadas a los bajos valores de $\mathrm{pH}$, que producen efectos negativos sobre la fisiología de las especies, y que además pueden tener una influencia directa sobre la disponibilidad de recursos tróficos (MULHOLLAND et al., 1987). De hecho la acidificación reduce la calidad del detrito, y por lo tanto puede inducir la reducción de la diversidad de las comunidades bentónicas o de la densidad de las poblaciones de macroinvertebrados (GROOM \& HILDREW, 1989).

En este trabajo se ha analizado el proceso de descomposición de hojarasca correspondiente a dos especies vegetales en tres tramos del torrente llamado Fosso Caldara, con notables diferencias en los valores de $\mathrm{pH}$, que corresponden a un gradiente natu- ral. La descomposición de hojarasca ha sido estudiada considerando que dicho detrito es un recurso trófico fundamental para la comunidad de macroinvertebrados lóticos (CUMMINS et al., 1974; BIRD \& KAUSHIK, 1987; FANO et al., 1988, 1991).

\section{ÁREA DE ESTUDIo}

El Fosso Caldara nace en la proximidad de la azufrera llamada Fontanile Nuovo (La Caldara) próxima a Roma, al Norte de la Región del Lacio (Italia Central) (fig. 1). En esta azufrera se producen vapores de $\mathrm{CO}_{2}$ y de $\mathrm{H}_{2} \mathrm{~S}$ que emergen a través de rocas piroclásticas, que pertenece a la Cadena Volcánica Sabatina (DEVOTO \& LOMBARDI, 1977). La longitud del torrente Fosso Caldara es de 7,5 Km. Se une con otros torrentes (Fosso della Mola, Fosso Pianciano, Fosso Vaccinella) con los cuales da origen al Fosso Vaccina, que desemboca en el Mar Tirreno (fig. 1).

Para este estudio fueron escogidas tres estaciones de muestreo (fig. 1). Las estaciones de muestreo 1 y 2 están aproximadamente a $1,5 \mathrm{Km}$ de distancia, mientras las estaciones 2 y 3 están aproximadamente a $4 \mathrm{Km}$.

En la Tabla 1 se muestran varias características fisico-químicas de las estaciones estudiadas. Se observan importantes dife- 


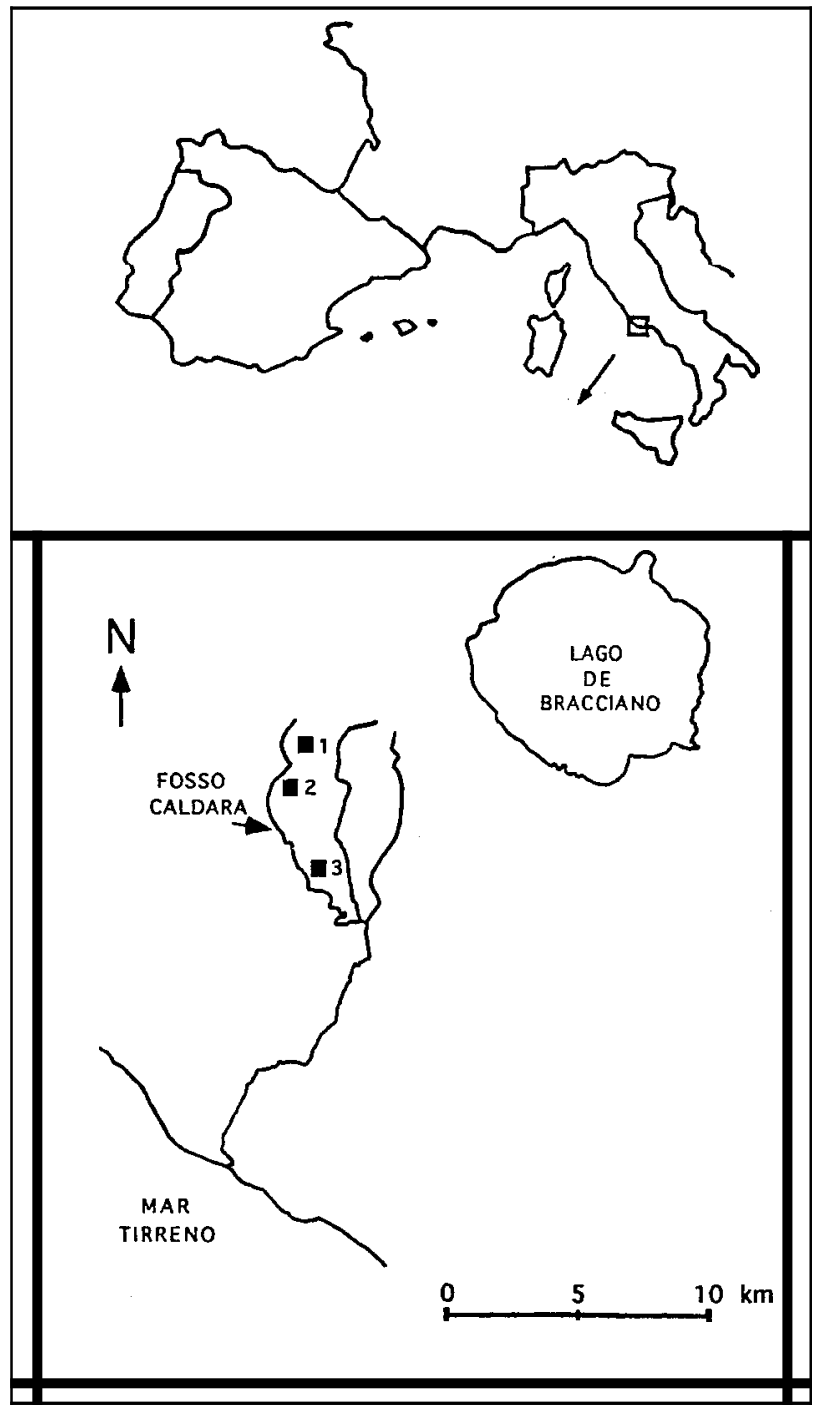

FIGURA 1. Área de estudio. Estaciones de muestreo. FIGURE 1. Study area. Sampling sites.

rencias entre estaciones para los valores de $\mathrm{pH}$ y acidez aguas abajo, desde condiciones de alta acidez en la estación 1 hasta valores próximos a la neutralidad en la estación 3.
Las tres estaciones de estudio se encuentran en un tramo fluvial de $1^{\text {er }}$ orden (VANNOTE et al., 1980). La vegetación ribereña es abundante, representada sobre todo por encinas, castaños, alisos, y abedules.

\section{MATERIAL Y MÉTODOS}

Treinta paquetes de hojarasca $\left(10 \mathrm{~cm} \mathrm{x} 10 \mathrm{~cm}, 0.5 \mathrm{~cm}^{2}\right.$ de malla) de aliso (Alnus glutinosa) y treinta de castaño (Castanea sativa) de $8 \mathrm{~g}$ de peso seco fueron colocados en el fondo del río en cada estación de muestreo (total $=180$ paquetes en las 3 estaciones). Los paquetes de hojarasca (5 de cada especie) fueron recogidos después de 7, 21, 35, 56, 77 y 105 días de inmersión, junto a muestras de agua utilizadas para la determinación de la temperatura, la concentración de oxígeno disuelto (método Winkler), la acidez (titulación con $\mathbf{H C l}$ ) y el $\mathbf{p H}$. La velocidad de la corriente, medida con correntímetro, es casi siempre mayor en la estación 3. La granulometría fue calculada pasando el sedimento a través de cedazos con mallas decrecientes, y calculando el peso seco de las fracciones obtenidas.

Los paquetes de hojarasca, llevados al laboratorio, fueron lavados y secados $\left(60^{\circ} \mathrm{C}, 72 \mathrm{~h}\right)$ para obtener el peso seco, y colocados en horno-mufla $\left(800^{\circ} \mathrm{C}, 4 \mathrm{~h}\right)$ para obtener el peso de las cenizas. Se calcularon los valores de biomasa mediante la diferencia de dichos valores (peso seco libre de cenizas: "AFDW= ash free dry weight"), con los cuales se calculó el coeficiente $\mathbf{k}$ de descomposición de los paquetes de hojarasca (PETERSEN \& CUMMINS, 1974): $\mathrm{k}=-\ln (\% \mathrm{R} / 100) \cdot \mathrm{t}^{-1}$, considerando que $\mathrm{R}$ es el peso del material remanente y t es el tiempo transcurrido expresado en días. El valor numérico del coeficiente $\mathbf{k}$ indica la velocidad de descomposición de las hojas: $\mathbf{k}<0.005$ es lenta; $0.005<\mathrm{k}<0.010$ es media; k>0.010 es rápida, según PETERSEN \& CUMMINS (1974).

Los valores de los coeficientes $\mathrm{k}$ han sido comparados mediante el test ANOVA para evidenciar las diferencias interespecíficas de descomposición de hojarasca para una misma localidad y diferencias intraespecíficas entre las tres estaciones.

Los macroinvertebrados que colonizaron los paquetes de hojarasca fueron identificados por taxones y por grupos tróficos

TABLA 1. Características físico-químicas del agua y granulometna del substrato en las tres estaciones de muestreo. Se muestran los valores mínimos y máximos. TABLE 1. Physico-chemical features of water and substrate granulometric fractions in the three sampling sites. Maxima and minima values are showed.

\begin{tabular}{|c|c|c|c|c|c|c|c|}
\hline Estación & $\mathbf{p H}$ & $\operatorname{acidez}^{*}$ & $\mathrm{O}_{2}$ & temperatura & velocidad & profundidad & granulom. \\
\hline & & $\mathrm{ppm} \mathrm{CaCO}_{3}$ & ppm & ${ }^{\circ} \mathrm{C}$ & $\mathbf{m} / \mathbf{s}$ & $\mathbf{m}$ & $\mathbf{m m}$ \\
\hline 1 & $2.15-2.88$ & $175-340$ & $9.7-9.8$ & $4-12.3$ & $0.01-0.22$ & $0.20-0.25$ & $1-5$ \\
\hline 2 & $3.25-3.74$ & $20-70$ & $12-12.5$ & $6.3-12.3$ & $0.01-0.28$ & $0.25-0.30$ & $5-10$ \\
\hline 3 & $6.70-7.72$ & $10-25$ & $11.1-12.3$ & $6.9-13$ & $0.22-0.33$ & $0.30-0.35$ & $0.5-1$ \\
\hline
\end{tabular}


funcionales (ANDERSON \& SEDELL, 1979, modificado). Se calculó la proporción de cada taxón y los índices de colonización ( $\mathrm{n}^{\circ}$ medio de individuos/paquete de hojarasca), y los coeficientes de diversidad trófica (HILDREW et al., 1984).

Se calculó también el índice de similaridad (SORENSEN, 1948) comparando los taxones encontrados en los paquetes de hojarasca de la misma especie (aliso o castaño) colocados en cada estación de muestreo: $(\mathrm{S})=2 \mathrm{Cl}(\mathrm{A}+\mathrm{B})$, donde $\mathrm{A}=$ número total de taxones en la estación "A"; $\mathrm{B}=$ número total de taxones en la estación "B"; $\mathrm{C}=$ número de taxones en común entre las estaciones"A" y "B".

\section{RESULTADOS}

Las figuras 2 y 3 muestran la dinámica de la pérdida de peso de los paquetes de hojarasca duranteel período del experimento en las tres estaciones de muestreo, para las dos especies vegetales Alnus glutinosa y Castanea sativa respectivamente.

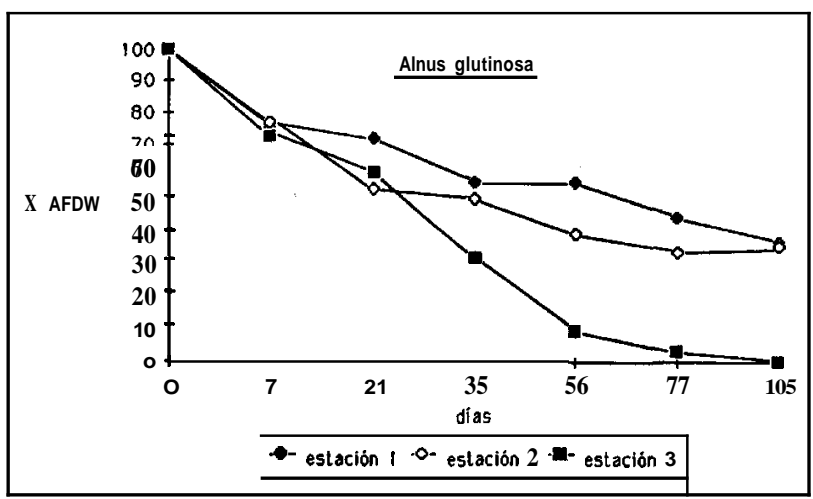

FIGURA 2. Descomposición de paquetes de hojarasca de Alnus glutinosa en las tres estaciones de muestreo.

FIGURE 2. Processing of Alnus glutinosa leaf packs in the 3 sampling sites.

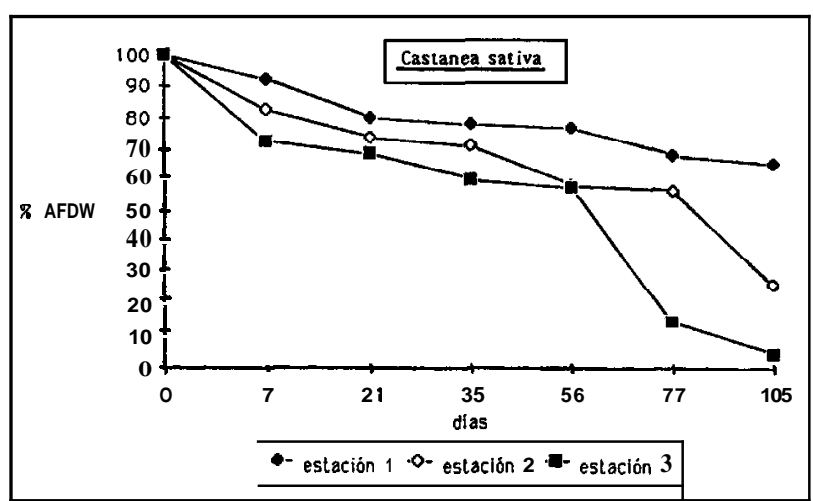

FIGURA 3. Descomposición de paquetes de hojarasca de Castanea sativa en las tres estaciones de muestreo.

FIGURE 3. Processing of Castanea sativa leaf packs in the 3 sampling sites.
En la estación de muestreo 1 el proceso de descomposición de la hojarasca de aliso (Alnus glutinosa) fue de velocidad media $(\mathrm{k}=0.0092)$, mientras que el de castaño (Castanea sativa) fue de velocidad lenta $(\mathrm{k}=0.0040)$; en la estación de muestreo 2 la velocidad de descomposición fue media $(\mathrm{k}=0.095)$ para $\mathrm{A}$ glutinosa, mientras que fue rápida $(\mathrm{k}=0.0147)$ para $\mathrm{C}$. sativa; en la estación 3 el proceso de descomposición de la hojarasca de las dos especies fue rápido, de hecho el coeficiente $\mathrm{k}$ de Alnus glutinosa $=0.0773$, mientras que el coeficiente $\mathrm{k} \mathrm{de}$ Castanea sativa $=0.0409$.

La Tabla 2 muestra los resultados del test ANOVA sobre los coeficientes k de descomposición de las dos especies de hojarasca.

TABLA 2. Comparación entre los valores del coeficiente $\mathbf{k}$ de descomposición de hojarasca de Alnus glutinosa y de Castanea sativa en las estaciones de muestreo $(1,2,3)$ (test ANOVA, $\left.{ }^{*}=\mathrm{p}<0.05\right)$.

TABLE 2. Comparision between k values of detritus processing of Alnus glutino$s a$ and Castanea sativa leaf packs collected at each sampling site $(1,2,3)$ (test ANOVA, ${ }^{*}=\mathrm{p}<0.05$ ).

Alnus 1 Alnus 2 Alnus 3 Castanea 1 Castanea 2 Castanea 3

Alnus 1

Alnus 2

Alnus 3

Castanea 1

Castanea 2

Castanea 3

La comparación entre los valores del coeficiente $\mathrm{k}$ de las dos especies de hojarasca en las mismas estaciones evidencia que existe una diferencia interespecífica significativa $(\mathrm{p}<0.05)$ sólo en la estación 3.

La comparación entre los valores del coeficiente $\mathrm{k}$ de hojarasca de Alnus glutinosa en cada una de las tres estaciones muestra que hay una diferencia intraespecífica significativa $(\mathrm{p}<0.05)$ entre las estaciones 1 y 3 , y entre las estaciones 2 y 3 . La comparación entre los valores del coeficiente $\mathrm{k}$ de Castanea sativa indica una diferencia significativa sólo entre las estaciones 1 y 3.

Las Tablas 3 y 4 muestran las proporciones de los taxones encontrados en los paquetes de hojarasca de Alnus glutinosa y Castanea sativa. Los taxones que presentan mayores proporciones son los quironómidos (en ambos paquetes de hojarasca de la estación 1) y los plecópteros Leuctra sp. (en los paquetes de $\boldsymbol{A}$. glutinosa de las estaciones 2 y 3 , y en los paquetes de $\mathrm{C}$. sativa de la estación 2) y Nemoura sp. (en los paquetes de C. sativa de la estación 3). Los grupos tróficos mayormente representados en los paquetes de hojarasca son el grupo de recolectores filtradores en la estación 1, y el grupo de los invertebrados fragmentadoresen las estaciones 2 y 3 . 
TABLA 3. Proporciones de los taxones de cada gmpo trofico encontrados en los paquetes de hojarasca de Alnus glutinosa en las $\mathbf{3}$ estaciones.

$\mathrm{Sh}=$ fragmentadores, $\mathrm{GIC}=$ recolectores no filtradores, $\mathrm{F} / \mathrm{C}=$ recolectores filtradores, $\mathrm{Sc}=$ raspadores, $\mathrm{Pr}=$ depredadores.

Se muestran además los valores de similaridad entre dichas estaciones.

TABLE 3. Frequencies of each taxon and trophic groups found on Alnus glutinosa leaf packs at the 3 sampling sites.

$\mathrm{Sh}=$ shredder, $\mathrm{G} / \mathrm{C}=$ gathering collector, FIC $=$ filtering collector, $\mathrm{Sc}=$ scraper $\operatorname{Pr}=$ Predators.

Similarity index values are also showed.

\begin{tabular}{|c|c|c|c|c|}
\hline TAXON & GR. TROF. & EST- 1 & EST-2 & EST-3 \\
\hline Leuctra sp. & Sh & & .760 & .536 \\
\hline Nemoura sp. & Sh & & & .222 \\
\hline Gammaridae & Sh & & & .010 \\
\hline Helodidae & Sh & .333 & & \\
\hline Limnephilidae & Sh & & & .005 \\
\hline Trichoptera n.c. & Sh & & .003 & \\
\hline Tipulidae & Sh & & & .005 \\
\hline Dryopidae & Sh & & & .005 \\
\hline TOTALSh & & .333 & .763 & .783 \\
\hline Baetis rhodani & GIC & & & .005 \\
\hline Plectrocnemia sp. & $\mathbf{G} / \mathbf{C}$ & & .005 & .005 \\
\hline TOTAL G/C & & & .005 & .010 \\
\hline Chironomidae & $\mathbf{F} / \mathrm{C}$ & .667 & .196 & .186 \\
\hline TOTAL F/C & & .667 & .196 & .186 \\
\hline TOTAL $(\mathrm{G}+\mathrm{F}) / \mathrm{C}$ & & .667 & .201 & .196 \\
\hline Odontocerum albicorne & Sc & & & .005 \\
\hline Planorbis sp. & Sc & & & .005 \\
\hline TOTALSc & & & & .010 \\
\hline Rhyacophyla sp. & Pr & & & .005 \\
\hline Sialis sp. & Pr & & .008 & \\
\hline Ceratopogonidae & Pr & & .028 & \\
\hline Dytiscidae & Pr & & & .005 \\
\hline TOTAL Pr & & & .036 & .010 \\
\hline
\end{tabular}

TABLA 5. Características de las asociaciones de macroinvertebradosque colonizan los paquetes de hojas de Alnus glutinosa y Castanea sativa en las $\mathbf{3}$ estaciones de muestreo.

TABLE 5. Features of macroinvertebrates associations of Alnus glutinosa and Castanea sativa leaf packs in the 3 sampling sites.

\begin{tabular}{lclll}
\hline ÍNDICE & HOJA & EST 1 & EST2 & EST3 \\
\hline N" de taxones & A. glutinosa & 2 & 6 & 13 \\
& C. sativa & 3 & 7 & 8 \\
No total de individuos & A. glutinosa & 18 & 388 & 194 \\
& C. sativa & 30 & 375 & 153 \\
Colonización & A. glutinosa & 1.29 & 32.50 & 16.17 \\
(n' med. ind./paq.) & C. sativa & 2.50 & 31.25 & 12.75 \\
Diversidad trófica & A. glutinosa & 0.636 & 0.672 & 0.684 \\
& C. sativa & 0.639 & 0.695 & 0.638 \\
\hline \multirow{2}{*}{ Coeficiente de similitud } & A. glutinosa & 0.25 & 0.31 & 0.13 \\
(Sorensen) & C. sativa & 0.40 & 0.53 & 0.1 \\
\hline
\end{tabular}

TABLA 4. Proporciones de los taxones de cada gmpo trofico encontrados en los paquetes de hojarascade Castanea sativa en las $\mathbf{3}$ estaciones.

$\mathrm{Sh}=$ fragmentadores, $\mathrm{G} / \mathrm{C}=$ recolectores no filtradores, $\mathrm{F} / \mathrm{C}=$ recolectores filtradores, $\mathrm{Sc}=$ raspadores, $\mathrm{Pr}=$ depredadores.

Se muestran además los valores de similaridad entre dichas estaciones.

TABLE 4. Frequencies of each taxon and trophic groups found on Castanea sati$\boldsymbol{v} \boldsymbol{a}$ leaf packs at the 3 sampling sites.

$\mathrm{Sh}=$ shredder, $\mathrm{G} / \mathrm{C}=$ gathenng collector, $\mathrm{F} / \mathrm{C}=$ filtering collector, $\mathrm{Sc}=$ scraper, $\operatorname{Pr}=$ Predators. Similarity index values are also showed.

\begin{tabular}{|c|c|c|c|c|}
\hline TAXON & GR. TROF. & EST- 1 & EST-2 & EST-3 \\
\hline Leuctra sp. & Sh & & .789 & .333 \\
\hline Nemoura sp. & Sh & & .023 & .412 \\
\hline Gammaridae & Sh & & & .007 \\
\hline Helodidae & Sh & .100 & & \\
\hline Limnephilidae & Sh & & & .007 \\
\hline TOTALSh & & .100 & .812 & .759 \\
\hline Haplotaxidae & $\mathbf{G} / \mathbf{C}$ & & .005 & .007 \\
\hline Ceratopogonidae & $\mathrm{G} / \mathrm{C}$ & .100 & .016 & \\
\hline Tipulidae & $\mathbf{G} / \mathbf{C}$ & & & .007 \\
\hline Plectronemia sp. & G/C & & .003 & \\
\hline TOTAL GIC & & .100 & .024 & .014 \\
\hline Chironomidae & $\mathrm{F} / \mathrm{C}$ & .800 & .152 & .222 \\
\hline TOTAL F/C & & .800 & .152 & .222 \\
\hline TOTAL $(\mathrm{G}+\mathrm{F}) / \mathrm{C}$ & & .900 & .176 & .236 \\
\hline Sialis sp. & $\operatorname{Pr}$ & & .005 & \\
\hline Tabanidae & Pr & & & .007 \\
\hline TOTAL Pr & & & .005 & .007 \\
\hline
\end{tabular}

La Tabla 5 muestra varias características de las asociaciones de macroinvertebrados que colonizan los paquetes de hojarasca de Alnus glutinosa y Castanea sativa. En ambos tipos de paquetes se observaron densidades mayores de taxones en la estación 3, y mayores densidades de individuos en la estación 2 . Los valores del índice de colonización son mayores en la estación 2 , mientras que los valores del coeficiente de diversidad trófica muestran valores muy similares en todas las estaciones de muestreo. La mayor similitud en composición de taxones que colonizan el detrito se observa entre las estaciones de muestreo más cercanas (estaciones 1 y 2 , y estaciones 2 y 3 ). Por contra, la menor similitud entre las estaciones que se encuentran a mayor distancia (estaciones 1 y 3 ). Se puede observar, de todos modos, que tales valores no son elevados (Tabla 5). La figura 4 muestra el número medio de individuos y de fragmentadores observados en cada muestreo en los paquetes de hojarasca de aliso y de castaño.

\section{DISCUSIÓN}

Los resultados obtenidos han evidenciado que la acidez del agua reduce la velocidad de descomposición del detrito. De hecho, sólo en condiciones de pH próximo a la neutralidad (esta- 


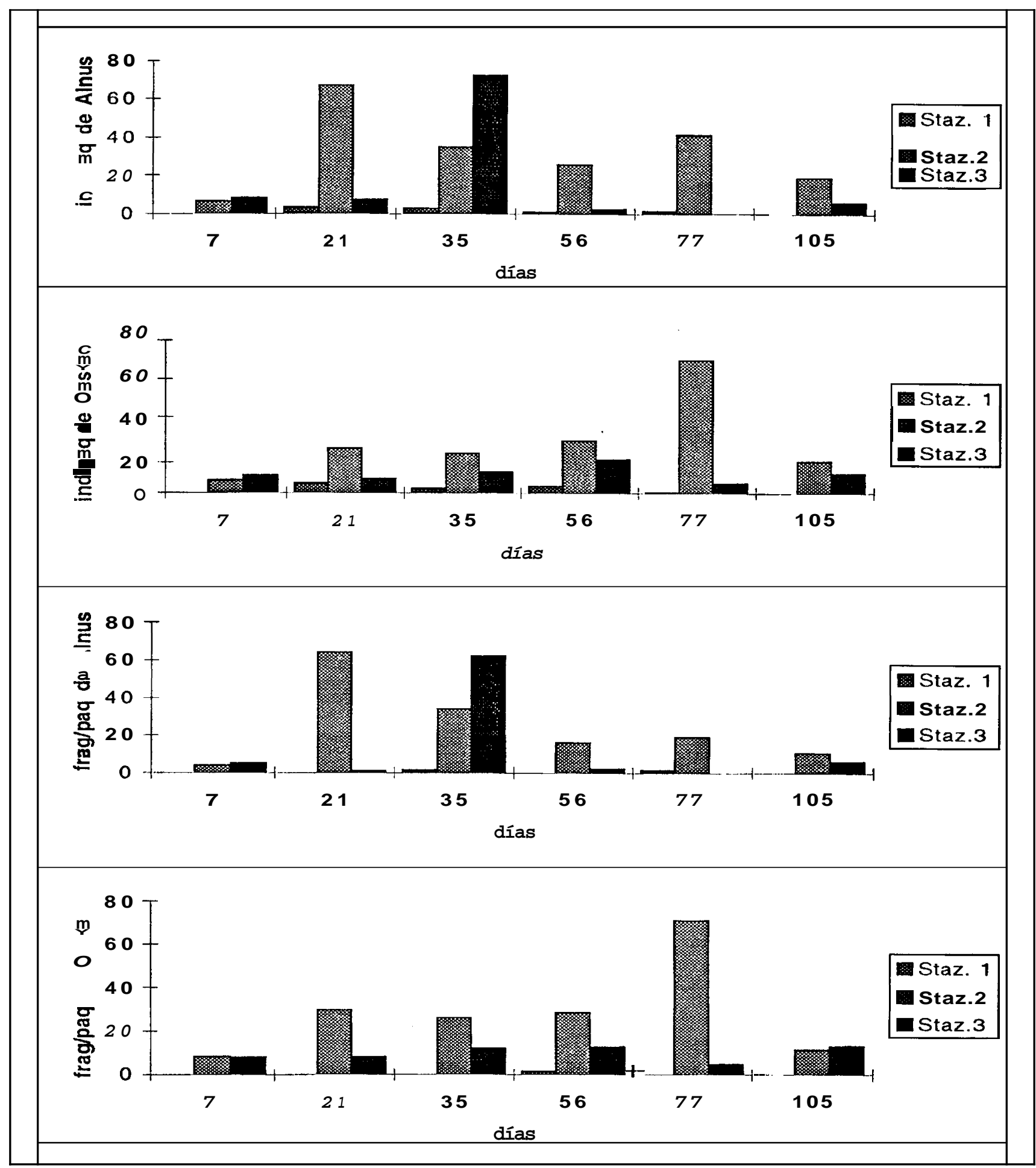

FIGURA 4. Númeromedio de individuoslpaquetede hojarasca y número de fragmentadoreslpaquetede hojarasca de cada muestreo en las 3 estaciones. FIGURE 4. Mean number of individuals/leaf pack and shredders/leaf pack for every sampling at each sampling site. 
ción de muestreo 3) es posible observar un valor del coeficiente $\mathrm{k}$ de descomposición comparable con los valores obtenidos en otras investigaciones realizadas con paquetes de las mismas hojas (FANO et al., 1981; FANO \& ROSSI, 1982). Estos resultados son similares a los que se han obtenido en otras investigaciones, que han evidenciado que en condiciones de acidez la utilización del detrito como recurso energético se reduce fundamentalmente por su baja calidad (HILDREW et al., 1984; PALUMBO et al., 1987; GROOM \& HILDREW, 1989) .

Varios autores han demostrado que la elevada acidez del agua, así como las condiciones químicas asociadas, mayor concentración de ciertos iones presentes en el agua como aluminio, hierro y zinc, ejercen un efecto negativo sobre la actividad de los hongos y de las bacterias durante la fase inicial de colonización del detrito (PALUMBO et al., 1987). De hecho, ha sido evidenciado que la cantidad de hongos y bacterias que colonizan el detrito en ambiente ácido es inferior (CHAMIER, 1987), así como la actividad de descomposición (PALUMBO et al., 1987; GROOM \& HILDREW, 1989).

La baja calidad del detrito que se observa en ambiente ácido influye además sobre la comunidad de macroinvertebrados que logra colonizar dicho recurso trófico. En este trabajo se ha evidenciado que en las estaciones donde hay mayor acidez del agua, la comunidad de macroinvertebrados es menos rica en taxones.

La diferencia significativa que se observa en la estación de muestreo 3 entre los valores del coeficiente de descomposición k de Alnus glutinosa y Castanea sativa se debe probablemente a la mayor frecuencia de los taxones del grupo de fragmentadores en los paquetes de hojarasca de Alnus glutinosa. La preferencia de los fragmentadores por las hojas de aliso sobre las de castaño se puede deber a la mayor concentración de nitrógeno que tienen las hojas de aliso respecto a las hojas de otras especies arbóreas de las regiones templadas y boreales (CHAPIN \& KEDROWSKI, 1983), y además por la baja concentración de taninos (IRONS et al., 1988).

Si se compara la descomposición del mismo tipo de hojarasca en diferentes condiciones de acidez se puede observar que en la estación 3 (pH próximo a la neutralidad) la hojarasca de castaño se descompone a una velocidad significativamente diferente a la que se observa en la estación 1 (pH más ácido) (Tabla 2), mientras que la velocidad de descomposición de la hojarasca de aliso en la estación 3 se diferencia no sólo a la que se observa en la estación 1, sino también a la de la estación 2 ( $\mathrm{pH}$ intermedio). Este resultado se puede relacionar con la diferente textura que tienen las dos especies de hojarasca. De hecho la de Castanea sativa tiene una textura que ofrece mayor resistencia a las variaciones de pH que la de Alnus glutinosa, y sólo en condiciones de elevada acidez se descompone a una velocidad significativamente diferente.

El grupo trófico más frecuente en la descomposición de los paquetes de hojarasca de $\boldsymbol{A}$. glutinosa y $\boldsymbol{C}$. sativa ha sido el grupo de los fragmentadores. La dominancia de dicho grupo, junto al grupo de depredadores, ha sido también observada por GROOM \& HILDREW (1989) en algunos ríos ácidos ingleses.

La Tabla 4 muestra las mayores frecuencias de los taxones de este grupo trófico en las estaciones 2 y 3, entre las cuales predominan las de los plecópteros Leuctra sp. y Nemoura sp. La dominancia de los fragmentadores se puede relacionar con la mayor velocidad de descomposición observada en dichas estaciones de muestreo. De hecho, los taxones de este grupo descomponen activamente el detrito CPOM ("coarse particulate organic matter, diámetro > $1 \mathrm{~mm}$ ) (CUMMINS et al., 1989), o sea los paquetes de hojarasca utilizados en este estudio. La relación entre la velocidad de descomposición del detrito y la acción de los fragmentadores se ha observado también en un estudio sobre la descomposición de paquetes de hojarasca de encina, efectuado en el río Arrone (FANO et al.,1991), situado en una zona próxima al Fosso Caldara. En nuestro estudio, no se observó ninguna diferencia significativa (ANOVA, $\mathrm{p}>0.05$ ) entre el número medio de fragmentadores por paquete de hojarasca de aliso y de castaño en las mismas estaciones de muestreo.

Solamente en la estación de muestreo 3 ha sido posible observar taxones pertenecientes al grupo trófico de los raspadores (Tabla 2). La ausencia de tal grupo en ambiente ácido (estaciones 1 y 2) se puede explicar con la escasa presencia de especies vegetales que se observa normalmente en ambientes lóticos ácidos (VAN DAM, 1988).

En la estación de muestreo 3 (condiciones de circum-neutralidad del agua) se observó que los valores del coeficiente k de descomposición resultan ser más elevados que en las estaciones 1 y 2 . En dicha estación, la comunidad de macroinvertebrados, más diversificada y rica puede influenciar positivamente la velocidad del proceso de degradación del detrito, contrariamente a lo que se observa en ambiente ácido (estaciones 1 y 2).

Los taxones en los dos tipos de paquetes de hojarasca no han sido siempre los mismos. De hecho, Planorbis sp., Dytiscidae, Rhyacophyla sp., Dryopidae, Odontocerum albicome, $y$ el efemeróptero Baetis rhodani han sido encontrados sólo en los paquetes de A. glutinosa, mientras Haplotaxidae y los dípteros Tabanidae han sido observados sólo en los paquetes de $\boldsymbol{C}$. sati$\boldsymbol{v} \boldsymbol{a}$. Esta diferencia de colonización demuestra que el detrito ejerce una selección de taxones, aunque el número medio de individuoslpaquete no ha sido significativamente diferente (ANOVA, $p>0.05$ ). En fin, los valores del coeficiente de similitud (S) indican que en un gradiente de acidez ácido-neutro, co- 
mo el que se observa en el torrente Fosso Caldara, las asociaciones de taxones que colonizan los paquetes de hojarasca se asemejan mayormente entre las estaciones de muestreo más cercanas, que presentan valores similares de acidez del agua.

\section{BIBLIOGRAFÍA}

ANDERSON, N. H. \& J. R. SEDELL, 1979. Detritus processing by macroinvertebrates in stream ecosystems. Ann. Rev. Entomol., 24: 351-377.

BIRD, G. A. \& N. K. KAUSHIK, 1987. Processing of maple leaf, grass and fern packs and their colonization by invertebrates in a stream . J. Freshwater Ecol. , 4 (2): 177-189.

CHAMIER, A.C., 1987. The effect of $\mathrm{pH}$ on microbial degradation of leaf litter in seven streams in the English Lake District. Oecologia , 71: 491-500.

CHAPIN, F. S. \& R.A. KEDROWSKI, 1983. Seasonal changes in nitrogen and phosphorus fractions and autumn translocation in evergreen and decidous taiga trees. Ecology, 64:376-391.

CUMMINS, $\boldsymbol{K}$. W., R. PETERSEN, F. O. HOWARD, J. C. WUYCHECK \& V. I. HOLT, 1974. The utilization of leaf litter by stream detritivores. Ecology, 54: 336-345.

CUMMINS, K. W., M. A. WILZBACK, D. M. GATES, J. B. PERRY \& W. B. TALIAFERRO, 1989. Shredders and riparian vegetation. Bioscience , 39 (1): 24-30.

DEVOTO, G. \& G. LOMBARDI, 1977. Le formazioni sedimentarie ed eruttive del settore Tolfetano- Cerite- Manziate (Lazio nordoccidentale). Accademia Nazionale dei Lincei, 227: 5-35.

FANO, E. A., L. ROSSI \& A. BASSET, 1981. Processo di demolizione del detrito vegetale alloctono e comunità ad esso associate in un corso d'acqua dell'Italia centrale. Atti S.IT.E. ,243-248.

FANO, E. A. \& L. ROSSI, 1982. Early stages of leaf detritus processing in the Garigliano river. Accademia Nazionale dei Lincei, Vol. LXXII: 158-162.

FANO, E. A., L. CASTELNUOVO, M. COLANGELO, E. MARCHETTI, J. G. MORGANA, G. PUPPI \& M. ZAMORANI, 1988. Decomposition process of Quercus ilex leaves in Mignone River (Italy). Boll. Mus. St. Nat. Lunigiana, 67:127-131.
FANO, E. A., M. ZAMORANI \& J. G. MORGANA, 1991. Procesos de descomposición y macroinvertebrados asociados en un río italiano. Limnética, 7:61-69.

GROOM, A. P. \& A. G. HILDREW, 1989. Food quality for detritivores in streams of contrasting pH. Journal of Animal Ecology, 58: 863-881.

HILDREW, A. G., C. R. TOWNSEND, J. FRANCIS \& K. FINCH , 1984. Cellulolytic decomposition in streams of contrasting $\mathrm{pH}$ and its relationships with macroinvertebrate community structure. Freshwater biology, 14: 323-328.

IRONS , J.G., M.W. Oswood \& J.P. Bryant , 1988. Consumption of leaf detritus by a stream shredder: Influence of tree species and nutrient status.Hydrobiologia, 160: 5361.

KIRBY, J. M. 1992. Red maple (Acerrubrum) leaf decomposition in an acid-impacted stream in North Central Pennsylvania. J. Freshwater Ecol. ,7 (1): 17-24.

MULHOLLAND, P. J., A. V. PALUMBO \& J. W. ELWOOD, 1987. Effects of acidification on leaf decomposition in streams. Journal of the North American Benthological Society ,6: 147-158.

PALUMBO, A. V., P. J. MULHOLLAND \& J. W. ELWOOD, 1987. Microbial communities on leaf material protected from macroinvertebrate grazing in acidic and circumneutral streams. Can. J. Fish. Aquat. Sci. ,44: 1064-1070.

PETERSEN, R. C. \& K. W. CUMMINS, 1974. Leaf processing in a woodland stream. Freshwater biology ,4: 343-368.

SORENSEN, T. 1948. A method of establishing groups of equal amplitude in plant society based on similarity of species content. K. Danske Vidensk. Selsk., 5: 1-34.

TOWNSEND, C. R., A. G. HILDREW \& J. E. FRANCIS, 1983. Community structure in some southern English streams: the influence of physico-chemical factors. Freshwater biology ,13: 521-544.

VAN DAM, H. 1988. Acidification of three moorland pools in the Netherlands by acid precipitation and extreme drought periods over seven decades. Freshwater Biology, 20: 157176.

VANNOTE, R. L., G. W. MINSHALL, K. W. CUMMINS, J. R. SEDELL \& C. E. CUSHING, 1980.The River Continuum Concept. Can. J. Fish. Aquat. Sci., 37: 130-137. 